\title{
STRICTLY SINGULAR PERTURBATION OF ALMOST SEMI-FREDHOLM LINEAR RELATIONS IN NORMED SPACES
}

\author{
T. ÁLVAREZ \\ Department of Mathematics, University of Oviedo, C/Calvo Sotelo $s / n$, \\ Oviedo 33007, Asturias, Spain \\ e-mail:seco@uniovi.es
}

(Received 23 July 2012; revised 24 January 2013; accepted 3 April 2013; first published online 13 August 2013)

\begin{abstract}
In this paper, we introduce the notions of almost upper semi-Fredholm and strictly singular pairs of subspaces and show that the class of almost upper semiFredholm pairs of subspaces is stable under strictly singular pairs perturbation. We apply this perturbation result to investigate the stability of almost semi-Fredholm multi-valued linear operators in normed spaces under strictly singular perturbation as well as the behaviour of the index under perturbation.
\end{abstract}

2000 Mathematics Subject Classification. 47A06.

1. Introduction. We recall some basic definitions and properties of linear relations in normed spaces following the notation and terminology of the book [7]. Let $X, Y$ and $Z$ denote infinite dimensional normed spaces over $\mathbb{K}=\mathbb{R}$ or $\mathbb{C}$. A linear relation or multi-valued linear operator $T$ from $X$ to $Y$ is a mapping from a subspace $D(T)=\{x \in$ $X: T x \neq \emptyset\}$, called the domain of $T$, into the collection of non-empty subsets of $Y$ such that $T\left(\alpha x_{1}+\beta x_{2}\right)=\alpha T x_{1}+\beta T x_{2}$ for all non-zero $\alpha, \beta$ scalars and $x_{1}, x_{2} \in D(T)$. The class of such linear relations is denoted by $L R(X, Y)$. If $T$ maps the points of its domain to singletons, then $T$ is said to be a single valued or simply an operator. A linear relation $T$ is uniquely determined by its graph, $G(T)$, which is defined by $G(T):=\{(x, y) \in X \times Y: x \in D(T), y \in T x\}$. It is easy to see that if $T \in L R(X, Y)$ then $D(T) \times Y=G(T)+(\{0\} \times Y)$ and $\{0\} \times T(0)=G(T) \cap(\{0\} \times Y)$.

Let $T \in L R(X, Y)$. The inverse of $T$ is the linear relation $T^{-1}$ given by $G\left(T^{-1}\right):=$ $\{(y, x):(x, y) \in G(T)\}$. The subspaces $T^{-1}(0):=N(T)$ and $R(T):=T(D(T))$ are called the null space and the range of $T$, respectively. We say that $T$ is injective if $N(T)=\{0\}$. We note that $D\left(T^{-1}\right)=R(T), D(T)=R\left(T^{-1}\right), T$ is single valued if and only if the subspace $T(0)$ coincides with $\{0\}$ and $y \in T x$ if and only if $T x=y+$ $T(0)$. We write $\alpha(T):=\operatorname{dim} N(T), \beta(T):=\operatorname{dim} Y / R(T), \bar{\beta}(T):=\operatorname{dim} Y / \overline{R(T)}, k(T):=$ $\alpha(T)-\beta(T)$, provided $\alpha(T)$ and $\beta(T)$ are not both infinite, and the topological index of $T$ is the quantity $\alpha(T)-\bar{\beta}(T)$, provided both $\alpha(T)$ and $\bar{\beta}(T)$ are not infinite.

For $S, T \in L R(X, Y)$ and $R \in L R(Y, Z)$ the sum $S+T$ and the product or composition $R S$ are defined by $G(S+T):=\left\{\left(x, y_{1}+y_{2}\right):\left(x, y_{1}\right) \in G(S),\left(x, y_{2}\right) \in\right.$ $G(T)\}$ and $G(R S):=\{(x, z):(x, y) \in G(S),(y, z) \in G(R)$ for some $y \in Y\}$.

Let $M$ and $N$ be subspaces of $X$ and the dual space $X^{\prime}$ respectively. Then $J_{M}$ denotes the natural injection map of $M$ into $X, M^{\perp}=\left\{x^{\prime} \in X^{\prime}: x^{\prime}(M)=0\right\}$, and if $M \cap D(T) \neq \emptyset$, then $\left.T\right|_{M}$ is given by $G\left(\left.T\right|_{M}\right):=\{(x, y) \in G(T): x \in M\}$ and $N^{\top}:=$ $\{x \in X: N(x)=0\}$. We observe that $\left.T\right|_{M} \neq T J_{M}$ but $\left.T\right|_{M}=T J_{M}$ if $T$ is single valued. 
The adjoint of $T \in L R(X, Y)$ is the linear relation $T^{\prime}$ defined by $G\left(T^{\prime}\right):=$ $G\left(-T^{-1}\right)^{\perp} \subset Y^{\prime} \times X^{\prime}$ and the reduced index of $T$ is the quantity $\bar{k}(T):=\alpha(\widetilde{T})-\alpha\left(T^{\prime}\right)$, provided both $\alpha(\widetilde{T})$ and $\alpha\left(T^{\prime}\right)$ are not infinite, where $\widetilde{T}$ denotes the linear relation defined by $G(\widetilde{T}):=\widetilde{G(T)} \subset \widetilde{X} \times \widetilde{Y}$ and $\widetilde{X}$ denotes the completion of $X$.

Let $T \in L R(X, Y)$. It is easy to see that if $Q_{T}$ denotes the quotient map from $Y$ onto $Y / \overline{T(0)}$, then $Q_{T} T$ is always a single-valued so that we may define $\|T x\|:=\left\|Q_{T} T x\right\|$, $x \in D(T)$ and $\|T\|:=\left\|Q_{T} T\right\|$ called the norm of $T x$ and of $T$ respectively. It follows from the definitions that $\|T x\|=d(y, T(0))$ for all $y \in T x$. We say that $T$ is continuous if $\|T\|<\infty$, open if its inverse is continuous equivalently if $\gamma(T)>0$, where $\gamma(T):=\infty$ if $D(T) \subset \overline{N(T)}$ and $\gamma(T):=\inf \{\|T x\| / d(x, N(T)): x \in D(T), x \notin \overline{N(T)}\}$ otherwise, almost open if $\lambda B_{R(T)} \subset \overline{T B_{X}}$ for some $\lambda>0$ equivalently if $T^{\prime}$ is open and $T$ is called closed if its graph is a closed subspace of $X \times Y$. We note that $T^{\prime}$ is always a closed linear relation, $T$ is closed if and only if $T^{-1}$ is closed, $T(0)$ is a closed subspace if $T$ is closed and we have a Closed Graph Theorem, that is, if $X$ and $Y$ are complete and $T \in L R(X, Y)$ is closed, then $T$ is open if and only if $R(T)$ is closed if and only if $T^{\prime}$ is open if and only if $R\left(T^{\prime}\right)$ is closed.

Let $T \in L R(X, Y)$. We say that $T$ is pre-compact if $Q_{T} T B_{X}$ is totally bounded, strictly singular, denoted by $T \in S S(X, Y)$ if there is no infinite dimensional subspace $M$ of $D(T)$ for which $\left.T\right|_{M}$ is injective and open, upper semi-Fredholm, denoted by $T \in F_{+}(X, Y)$ if there exists a finite co-dimensional subspace $M$ of $X$ such that $\left.T\right|_{M}$ is injective and open, and $\mathrm{T}$ is called lower semi-Fredholm, denoted by $T \in F_{-}(X, Y)$ if $T^{\prime} \in F_{+}\left(Y^{\prime}, X^{\prime}\right)$. We note the following useful properties:

$$
T \in F_{+} \Leftrightarrow T \in F_{-} \text {and } T \in F_{-} \Leftrightarrow T^{\prime} \in F_{+} .
$$

If $X$ and $Y$ are Banach spaces and $T$ is closed then $T \in F_{+} \Leftrightarrow T \in \phi_{+}$(that is, $R(T)$ is closed and $\alpha(T)<\infty)$ and $T \in F_{-} \Leftrightarrow T \in \phi_{-}$(that is, $R(T)$ is a closed finite co-dimensional subspace of $Y$ ).

(For the proofs see [7, Chapter V]).

Following [2, Definition 1] we say that $T$ is almost upper semi-Fredholm, denoted by $T \in A U S F(X, Y)$ if $T$ is open with finite dimensional null space, almost lower semiFredholm, denoted by $T \in A \operatorname{LSF}(X, Y)$ if $T$ is almost open and $\operatorname{dim} Y / \overline{R(T)}<\infty$, almost semi-Fredholm if $T \in A U S F(X, Y) \cup A L S F(X, Y)$ and $T$ is called an almost Fredholm linear relation if $T \in A U S F(X, Y) \cap A L S F(X, Y)$. We remark the following useful facts:

$A U S F \subset F_{+}$. In general, the inclusion is strict even for operators.

$A L S F=F_{-}$.

The classes $F_{+}$(resp. $F_{-}$) and $\phi_{+}$(resp. $\phi_{-}$) are in general different even for operators, but for closed linear relations between Banach spaces we have that $A U S F=$ $F_{+}=\phi_{+}$and $A L S F=F_{-}=\phi_{-}$.

Linear relations were introduced into Functional Analysis by von Neumann [19], motivated by the need to consider adjoints of non-densely defined linear differential operators, which are considered by Coddington and Dijksma [6] and Dikjsma et al. [8], among others. One main reason why linear relations are more convenient than operators is that one can define the inverse, the closure and the completion for a linear relation. Interesting works on multi-valued linear operators include the treatise on partial differential relations by Gromov [15], the application of multi-valued methods to the solutions of differential equations by Favini and Yagi [9], the development of fixed point theory for linear relations to the existence of mild solutions of quasi-linear 
differential inclusions of evolution and also to many problems of fuzzy theory (see, for instance $[\mathbf{1}, \mathbf{1 4}, \mathbf{1 8}, \mathbf{2 0}]$ ) and several papers on semi-Fredholm linear relations and other classes related to them (see, for instance [3-5]).

The purpose of this work is to investigate the stability of the $F_{+}, A U S F, F_{-}$and $A L S F$ linear relations in normed spaces under strictly singular perturbations, as well as the behaviour of the reduced index under perturbation. The results obtained generalize some pioneering work due to Kato [16] in the case of closed operators in Banach spaces.

In [3, Proposition 10], Álvarez began the study of the perturbation problem of closed $F_{+}$linear relations under strictly singular perturbation. More precisely, the author proves that if $T \in F_{+}(X, Y)$ is closed and $S \in S S(X, Y)$ is continuous such that $\overline{D(T)} \subset D(S)$ and $S(0) \subset T(0)$, then $T+S$ is a closed upper semi-Fredholm linear relation and $\bar{k}(T+S)=\bar{k}(T)$. This result will be applied in this paper to deduce the stability of closed lower semi-Fredholm linear relations under strictly singular perturbation (Theorem 10(ii)).

In [2] the almost semi-Fredholm linear relations in normed spaces are introduced and characterized in terms of nullity and deficiency. We note that two papers by Gheorghe $[\mathbf{1 0}, \mathbf{1 1}]$ have been devoted to the study of the perturbation of almost Fredholm linear relations under small and compact operator perturbations. In [11, Theorem 1], the author obtains necessary and sufficient conditions for the stability of the topological index of an almost Fredholm linear relation between normed spaces under small perturbations with linear relations, while in [10, Theorem 1] the author proves the following result.

THEOREM 1. Let $X, Y$ be normed spaces such that $Y$ is complete, and let $T \in$ $L R(X, Y)$ be an almost Fredholm linear relation. If $K$ is a bounded compact operator from $X$ to $Y$ then $\operatorname{dim} N(T+K)<\infty, \operatorname{dim} Y / \overline{R(T+K)}<\infty$, and if $T+K$ is open then $T+K$ and $T$ have the same topological index.

In this paper we show that Theorem 1 remains valid if $Y$ is an arbitrary normed space, $T$ is an almost semi-Fredholm linear relation and $K$ is a continuous strictly singular linear relation (Theorem 13). It is important to remark that the proofs of the results of Gheorghe ([10, Theorem 1], [11, Theorem 1]) are obtained using some techniques of the perturbation theory for quotient morphisms between normed spaces, and our proof of Theorem 13 is based on the concept of pairs of subspaces of a normed space. The method adopted here for the study of the perturbation of almost semi-Fredholm relations in normed spaces by means of pairs of subspaces seems to be new and emphasizes a strong connection between the perturbation theory of closed linear relations and that of some operators.

The paper is organized as follows: In Section 2 we define the almost upper semi-Fredholm and strictly singular pairs of subspaces and prove that the class of almost upper semi-Fredholm pairs of subspaces is stable under strictly singular pairs perturbation. This result is applied in Section 3 to analyse the perturbation of almost semi-Fredholm linear relations with strictly singular linear relations.

2. Strictly singular perturbation of almost semi-Fredholm pairs of subspaces of a normed space. The notions of classes of upper semi-Fredholm and strictly singular pairs of subspaces of a Banach space due to González [13, Definition 2.1] are generalized to normed spaces as follows. 
Definition 2. Let $M$ and $N$ be closed subspaces of a normed space $X$. We say that $(M, N) \in A U S F(\operatorname{resp} .(M, N) \in S S)$ if $Q_{N} J_{M} \in A U S F\left(\operatorname{resp} . Q_{N} J_{M} \in S S\right)$.

The aim of this section is to analyse the stability of the class of almost upper semi-Fredholm pairs of subspaces under strictly singular perturbation as well as the behaviour of the index under perturbation. For this end we need some auxiliary results

Lemma 3. Let $A$ be a closed subspace of $X$ and let $B \subset X$ be a subspace such that $A \subset B$. Then $B$ is closed if and only if $B / A$ is closed.

Lemma 4. ([17, Proposition 7]). Let $T$ be an operator from $X$ to $Y$ with finite dimensional null space. Then $T$ is open if and only if for every closed finite co-dimensional subspace $M$ of $D(T), T M$ is closed in $R(T)$ and $T J_{M}$ has a continuous inverse if $N(T) \cap$ $M=\{0\}$.

Lemma 5. ([7, Theorems V.1.6, V.2.4 and Corollary V.7.11]). Let $T \in L R(X, Y)$.

(i) $T$ is not upper semi-Fredholm if and only if there is no closed finite co-dimensional subspace $M$ of $X$ such that $\left.T\right|_{M}$ is injective and open if and only if there exists an infinite dimensional subspace $M$ of $D(T)$, (closed if $T$ is closed) such that $\left.T\right|_{M}$ is precompact.

(ii) $T$ is upper semi-Fredholm if and only if $\operatorname{dim} N(T+K)<\infty$ whenever $K \in$ $L R(X, Y)$ is precompact and $\operatorname{dim} K(0)<\infty$.

(iii) If $\operatorname{dim} D(T)=\infty$, then $T$ is upper semi-Fredholm if and only if $\Gamma(T)>0$, where $\Gamma(T):=\inf \left\{\left\|\left.T\right|_{M}\right\|: M\right.$ infinite dimensional subspace of $\left.D(T)\right\}$.

We have noted that $A U S F \subset F_{+}$and, in general, the inclusion is strict. However, as a consequence of Lemmata 4 and 5 we obtain the following useful result.

Lemma 6. Let $M$ and $N$ be closed subspaces of $X$. Then $Q_{N} J_{M} \in A U S F$ if and only if $Q_{N} J_{M} \in F_{+}$.

Proof. Assume that $Q_{N} J_{M} \in F_{+}$. By Lemma $5 \operatorname{dim} N\left(Q_{N} J_{M}\right)=\operatorname{dim}(M \cap N)<\infty$ and there exists a closed finite co-dimensional subspace $A$ of $D(T)$ such that $Q_{N} J_{A}$ has a continuous inverse.

Let us consider two possibilities for $Q_{N} J_{M}$ :

Case 1: $Q_{N} J_{M}$ is open. In such a case, $Q_{N} J_{M} \in A U S F$, as desired.

Case 2: $Q_{N} J_{M}$ is not open. Then by Lemma 4, $R\left(Q_{N} J_{A}\right)=N+A / N$ is not closed in $R\left(Q_{N} J_{M}\right)$ and thus it follows from the hypothesis together with Lemma 3 that $N+A$ is not closed in $X$ and equivalently $A^{\perp}+N^{\perp}$ is not closed in $X^{\prime}$, so that applying again Lemma 3 we infer that $A^{\perp}+N^{\perp} / A^{\perp}=R\left(Q_{A^{\perp}} J_{N^{\perp}}\right)$ is not closed. But, since $Q_{N} J_{A}$ is upper semi-Fredholm, we have that $\left(Q_{N} J_{A}\right)^{\prime}=Q_{A^{\perp}} J_{N^{\perp}} \in F_{-}$so that $Q_{A^{\perp}} J_{N^{\perp}} \in \phi_{-}$; in particular, $R\left(Q_{A^{\perp}} J_{N^{\perp}}\right)$ is closed, a contradiction.

Corollary 7. Let $M$ and $N$ be closed subspaces of $X$. Then $\operatorname{dim} M=\operatorname{dim} N=\infty$ if $(M, N) \notin A U S F$.

Proof. Assume $(M, N) \notin A U S F$. Then by Lemma $6, Q_{N} J_{M}$ is not upper semiFredholm. If $\operatorname{dim} M<\infty$, then it is obvious that $\{0\}$ is a closed finite co-dimensional subspace of $M$ and $Q_{N} J_{\{0\}}$ is an isometry, and hence $Q_{N} J_{M} \in F_{+}$, which is a contradiction with our assumption. If $\operatorname{dim} N<\infty$, then $Q_{N}$ is open with finite dimensional null space, and thus by virtue of Lemma 5 (iii), $\Gamma\left(Q_{N}\right)>0$, which implies that $\Gamma\left(Q_{N} J_{M}\right)>0$ so that again applying Lemma 5(iii) we conclude that $Q_{N} J_{M} \in F_{+}$, a contradiction. 
Proposition 8. Let $M$ and $N$ be closed subspaces of $X$.

(i) If $(M, N) \notin A U S F$, then there exists a precompact operator $K: X \rightarrow X$ and an infinite dimensional closed subspace $L$ of $M$ such that $\|K\|<1$ and $(I-K) L \subset$ $N$.

(ii) If there exists an everywhere defined precompact operator $K$ on $X$ and an infinite dimensional subspace $A$ of $M$ such that $(I-K) A \subset N$, then $(M, N) \notin A U S F$.

Proof. (i) Assume $(M, N) \notin A U S F$. In such a case $\operatorname{dim} M=\operatorname{dim} N=\infty$ by Corollary 7, and by Lemma 5(i) there is no closed finite co-dimensional subspace $B$ of $M$, for which $Q_{N} J_{B}$ is injective and open. Then by the Hahn-Banach theorem, a pair of sequences $\left(x_{n}\right) \subset M$ and $\left(x_{n}^{\prime}\right) \in X^{\prime}$ can be constructed as follows:

Select $x_{1} \in M$ and $y_{1} \in N$ such that $\left\|x_{1}-y_{1}\right\|<1 / 2$. Next, choose $x_{1}^{\prime} \in X^{\prime}$ such that $1=\left\|x_{1}\right\|=\left\|x_{1}^{\prime}\right\|=x_{1}^{\prime}\left(x_{1}\right)$.

Since $N\left(x_{1}^{\prime}\right)$ is closed and of co-dimension 1 in $X$, there exists $x_{2} \in M_{1}:=M \cap$ $N\left(x_{1}^{\prime}\right)$ and $y_{2} \in N$ such that $\left\|x_{2}\right\|=1$ and $\left\|x_{2}-y_{2}\right\|<1 / 2^{2}$. Next, choose $x_{2}^{\prime} \in X^{\prime}$ such that $1=\left\|x_{2}^{\prime}\right\|=x_{2}^{\prime}\left(x_{2}\right)$.

Continuing in this way, we obtain

$$
\left\|x_{k}\right\|=\left\|x_{k}^{\prime}\right\|=x_{k}^{\prime}\left(x_{k}\right)=1, y_{k} \in N,\left\|x_{k}-y_{k}\right\|<1 / 2^{k}, k \in \mathbb{N}
$$

and $x_{k} \in M_{k}:=M \cap N\left(x_{1}^{\prime}\right) \cap N\left(x_{2}^{\prime}\right) \cap \ldots . . . N\left(x_{k-1}^{\prime}\right)$ (so that $x_{i}^{\prime}\left(x_{k}\right)=0$ if $1 \leq i<k$ ).

These properties ensures that the set $\left\{x_{n}: n \in \mathbb{N}\right\}$ is linearly independent.

Let $A$ denote the subspace generated by sequence $\left(x_{n}\right)$, and let $P_{n}$ denote the projection defined on $M$ with range $M_{n}$ and null space the subspace generated by $\left\{x_{1}, x_{2}, \ldots, x_{n}\right\}$. Then $P_{n}$ is continuous and by virtue of the Hahn-Banach theorem we can find an element $f_{n} \in X^{\prime}$ such that $\left.f_{n}\right|_{M}=x_{n}^{\prime} P_{n-1}$, and moreover $f_{n}$ and $x_{n}^{\prime} P_{n-1}$ have the same norm. Hence, $f_{n}\left(x_{m}\right)=\delta_{n m}$ for all $1 \leq n \leq m$ and we can assume that $\left\|f_{n}\right\|\left\|x_{n}-y_{n}\right\|<1 / 2^{n}$ and $\left\|f_{n}\right\|<\left\|P_{n-1}\right\|$.

In this situation, we can consider the nuclear operator

$$
K: x \in X \rightarrow K x:=\sum_{n=1}^{\infty} f_{n}(x)\left(x_{n}-y_{n}\right) \in X .
$$

Clearly, $K$ is precompact and $\|K\|<1$. Moreover, $(I-K) x_{n}=y_{n}$ for each $n \in \mathbb{N}$. Thus, we can take as $L$ the closure of $A$.

(ii) If there exists an everywhere defined precompact operator $K$ on $X$ and an infinite dimensional subspace $A$ of $M$ such that $(I-K) A \subset N$, then $Q_{N}(I-K) J_{A}=0$ so that $Q_{N} J_{A}$ is precompact. Thus, the use of Lemmata 5(i) and 6 makes us conclude that $(M, N) \notin A U S F$. The proof is complete.

Proposition 8 generalizes Proposition 2.4(i) in [13, Proposition 2.4].

THEOREM 9. Let $L, M$ and $N$ be closed subspaces of $X$. If $(L, M) \in S S$ and $(M, N) \in$ $A U S F$ then $(L, N) \in A U S F$.

Proof. Suppose that $(L, M) \in S S$ and $(L, N) \notin A U S F$. By Proposition 8(i), there exists a precompact operator $K_{o}: X \rightarrow X$ with $\left\|K_{o}\right\|<1$ and an infinite dimensional closed subspace $L_{o}$ of $L$ such that $\left(I-K_{o}\right) L_{o} \subset N$.

On the other hand, $(L, M) \in S S$ so that $\left(L_{o}, M\right) \in S S$, and thus by Lemma 5(i), $\left(L_{o}, M\right) \notin F_{+}$. Thus, we infer from Lemma 6 that $\left(L_{o}, M\right) \notin A U S F$, and again Proposition 8(i) ensures that there exists an everywhere defined precompact operator $K_{1}$ on $X$ with $\left\|K_{1}\right\|<1$ and an infinite dimensional closed subspace $L_{1}$ of 
$L_{o}$ such that $\left(I-K_{1}\right) \subset M$. Since $\left\|K_{1}\right\|<1, I-K_{1}$ has a continuous inverse (see, for instance [12, Corollary V.1.3]) and so we can write $\left(I-K_{o}\right)\left(I-K_{1}\right)^{-1}:=I-K$, where $K$ is a bounded precompact operator on $X$.

Let us denote $M_{1}:=\left(I-K_{1}\right) L_{1}$. Now, since $(I-K) M_{1}=\left(I-K_{o}\right) L_{1} \subset N$, applying Proposition 8(ii), we get $(M, N) \notin A U S F$, which contradicts our assumption.

This Theorem 9 will play a crucial role in the proof of the result concerning the stability of the class $A U S F$ under strictly singular perturbations (Theorem 13).

3. Strictly singular perturbation of $F_{+}, F_{-}, A U S F$ and $A L S F$ linear relations. We start this section proving a result concerning the perturbation of closed $F_{+}$and $F_{-}$linear relations with strictly singular linear relations.

THEOREM 10. Let $T \in L R(X, Y)$ be closed and let $S \in L R(X, Y)$ be continuous such that $\overline{D(T)} \subset D(S)$ and $S(0) \subset T(0)$.

(i) If $T \in F_{+}(X, Y)$ and $S \in S S(X, Y)$, then $T+S$ is a closed $F_{+}$-relation and $\bar{k}(T+S)=\bar{k}(T)$.

(ii) If $T \in F_{-}(X, Y)$ and $S^{\prime} \in S S\left(Y^{\prime}, X^{\prime}\right)$, then $T+S$ is a closed $F_{-}$-relation and $\bar{k}(T+S)=\bar{k}(T)$.

Proof. We first note that $T+S$ is closed by [3, Lemma 7] and $(T+S)^{\prime}=T^{\prime}+S^{\prime}$ by [7, Proposition III.1.5].

(i) This statement was proved by Álvarez in [3, Proposition 10].

(ii) Since $S$ is continuous so is $S^{\prime}$ ([7, Corollary III.1.13]) and thus we infer from [7, Propositions III.1.4 and III.4.6] that

$\overline{D\left(T^{\prime}\right)} \subset\left(D\left(T^{\prime}\right)^{\top}\right) \perp=T(0)^{\perp} \subset S(0)^{\perp}=D\left(S^{\prime}\right) \quad$ and $\quad S^{\prime}(0)=D(S)^{\perp} \subset D(T)^{\perp}=$ $T^{\prime}(0)$, that is, $\overline{D\left(T^{\prime}\right)} \subset D\left(S^{\prime}\right)$ and $S^{\prime}(0) \subset T^{\prime}(0)$.

In this situation since $T^{\prime}$ is closed, we deduce from the part (i) applied to $T^{\prime}$ and $S^{\prime}$ that $T^{\prime}+S^{\prime}$ is a closed $F_{+}$-relation and $k\left(T^{\prime}\right)=k\left(T^{\prime}+S^{\prime}\right)$. The desired conclusion now follows upon noting that $\bar{k}(T)=-k\left(T^{\prime}\right)$ and $\bar{k}(T+S)=-k\left((T+S)^{\prime}\right)$ by virtue of [7, Proposition V.15.3].

Our next objective is to investigate the stability of the almost semi-Fredholm linear relations under strictly singular additive perturbation as well as the behaviour of the index under perturbation. Our investigation uses Theorem 9 in conjunction with some auxiliary properties.

LEMma 11. Let $S, T \in L R(X, Y)$ be closed such that $S$ is continuous, $\overline{D(T)} \subset D(S)$ and $S(0) \subset T(0)$. Then,

(i) the linear relation $U$ defined by

$$
G(U):=\{(x,(x, y)) \in X \times(X \times Y): x \in D(T),(x, y) \in G(S)\}
$$

is continuous, injective and open.

(ii) The linear relation $V: D(V):=D(T) \times Y / G(T) \subset X \times Y / G(T) \rightarrow Y / T(0)$ defined by

$$
V((x, y)+G(T)):=y-b+T(0),
$$

where $b \in Y$ is arbitrarily chosen with the property $(x, y)=(x, b)+(0, c)$ with $(x, b) \in G(T)$ is injective and open.

(iii) $S-T=V Q_{G(T)} J_{G(S)} U$. 
Proof. (i) It follows from the definitions that $U$ is an injective linear relation and $U(0)=\{0\} \times S(0)$.

We claim that

$$
\|U x\|=\|x\|+\|S x\| \text { forall } x \in D(T) .
$$

Indeed, let $x \in D(T)$. Then for all $(x, b) \in U x$ we have that $\|U x\|=$ $d((x, b), U(0))=d((x, b),\{0\} \times S(0))=\|x\|+d(b, S(0))=\|x\|+\|S x\|$, and hence (1) holds.

Now the property (1) combined with the continuity of $S$ and the injectivity of $U$ leads to $U$ is continuous and open.

(ii) We first show that $V$ is unambiguously defined. Let $(x, y) \in D(T) \times Y$, then $(x, y)$ can be decomposed as $(x, y)=\left(x, b_{1}\right)+\left(0, c_{1}\right)$ with $\left(x, b_{1}\right) \in G(T)$, and moreover if $(x, y)=\left(x, b_{2}\right)+\left(0, c_{2}\right)$ for some $\left(x, b_{2}\right) \in G(T)$, then $y-b_{1}+T(0)=$ $y-b_{2}+T(0)$, as desired.

That $V$ is injective follows trivially from the definitions, so that it only remains to see that $V$ is open. Let $(x, y) \in D(V)$. Then $(x, y)=(x, b)+(0, c)$ with $(x, b) \in G(T)$, which implies that

$$
\begin{gathered}
\|(x, y)+G(T)\|=d((x, y), G(T))=d((0, c), G(T))=d((0, y-b), G(T)) \\
\leq d((0, y-b),\{0\} \times T(0))=d(y-b, T(0))=\| V((x, y)+G(T) \| .
\end{gathered}
$$

Therefore, $V$ is open, as required.

(iii) Let $(x, y) \in S-T$. Then $(x, y)=\left(x, y_{1}-y_{2}\right)$ with $\left(x, y_{1}\right) \in G(S)$ and $\left(x, y_{2}\right) \in$ $G(T)$ so that $\left(x, y_{1}\right)=\left(x, y_{2}\right)+(0, y)$ and so we deduce from the definition of $V$ that

$$
V\left(\left(x, y_{1}\right)+G(T)\right)=y+T(0) .
$$

Furthermore, since $\left(x, y_{1}\right) \in G(S)$ and $x \in D(T)$, we have that

$$
Q_{G(T)} J_{G(S)}\left(x, y_{1}\right)=Q_{G(T)}\left(x, y_{1}\right)
$$

and thus by (2) we obtain that

$$
y+T(0)=V\left(\left(x, y_{1}\right)+G(T)\right)=V Q_{G(T)} J_{G(S)}\left(x, y_{1}\right) .
$$

Therefore, $S-T \subset V Q_{G(T)} J_{G(S)} U$.

Conversely, let $(x, y) \in V Q_{G(T)} J_{G(S)} U$. Then it is simple to show that there exists $\left(x, y_{1}\right) \in G(S)$ such that $y \in V\left(\left(x, y_{1}\right)+G(T)\right)=y_{1}-y_{2}+T(0)$, where $\left(x, y_{1}\right)=$ $\left(x, y_{2}\right)+(0, c)$ for $\left(x, y_{2}\right) \in G(T)$. Consequently, $y \in y_{1}-y_{2}+T(0)$ with $\left(x, y_{1}\right) \in G(S)$ equivalently $S x=y_{1}+S(0)$ and $\left(x, y_{2}\right) \in G(T)$ equivalently $T x=y_{2}+T(0)$ and since $S(0) \subset T(0)$, by hypothesis we conclude that $(S-T) x=y_{1}-y_{2}+T(0)$ and hence $y \in(S-T) x$, as required.

LEMMA 12. Let $T \in \operatorname{LR}(X, Y)$ be closed.

(i) If $T \in A U S F$, then $(X \times\{0\}, G(T)) \in A U S F$.

(ii) If $T \in S S$, then $(G(T), X \times\{0\}) \in S S$.

Proof. (i) Assume that $T \in A U S F$; in particular, $T$ is upper semi-Fredholm and thus there is a closed finite co-dimensional subspace $M$ of $X$ for which $\left.T\right|_{M}$ is injective and open. Let $L:=M \times\{0\}$. It is clear that $L$ is a closed finite co-dimensional subspace of $D\left(Q_{G(T)} J_{X \times\{0\}}\right)$ and $Q_{G(T)} J_{L}$ is injective. Hence, it only remains to verify that $Q_{G(T)} J_{L}$ is open. For this, let $z=(m, 0) \in L$. We have by a direct computation that the norm 
of $Q_{G(T)} J_{L}(m, 0)$ coincides with the norm of $T m$. Now the use of this last property combined with the fact that $\left.T\right|_{M}$ is injective and open makes us conclude that $Q_{G(T)} J_{L}$ is open, as required.

(ii) Assume that $(G(T), X \times\{0\}) \notin S S$, that is, $Q_{X \times\{0\}} J_{G(T)}$ is not strictly singular. Then there exists an infinite dimensional subspace $A$ of $D\left(Q_{X \times\{0\}} J_{G(T)}\right)=G(T)$ for which $Q_{X \times\{0\}} J_{A}$ is injective and open. Let $L:=\{x \in D(T):(z, y) \in A$ for all $y \in T z\}$. In order to show that $T$ is not strictly singular, it is enough to establish that $L$ is an infinite dimensional subspace of $D(T)$ such that $\left.T\right|_{L}$ is injective and open. That $L$ is an infinite dimensional subspace of $D(T)$ is obvious and that $N\left(\left.T\right|_{L}\right)=\{0\}$, it follows observing that if $z \in N\left(\left.T\right|_{L}\right)=N(T) \cap L$ then $0 \in T z$ with $z \in L$ so that $(z, 0) \in$ $A \cap X \times\{0\}=N\left(Q_{X \times\{0\}} J_{A}\right)=\{(0,0)\}$. Finally, we shall prove that $\left.T\right|_{L}$ is open. Let $z \in L$ and let $y \in T z$ be arbitrary but fixed. Then for all $b \in T(0)$ we have that $y-b \in$ $T z$ and $(z, y-b) \in A$ so that $\eta\|(z, y-b)\| \leq\left\|Q_{X \times\{0\}}(z, y-b)\right\|$ for some $\eta>0$ (as $Q_{X \times\{0\}} J_{A}$ is injective and open). In consequence, $\eta\|z\| \leq d(y, T(0))$, which implies that $\eta\|z\| \leq\|T z\|$ for some $\eta>0$, as desired. The proof is complete.

We are now ready to give the fundamental result of this paper.

THEOREM 13. Let $S, T \in L R(X, Y)$ be closed such that $S$ is continuous, $\overline{D(T)} \subset D(S)$ and $S(0) \subset T(0)$.

(i) If $T \in A U S F(X, Y)$ and $S \in S S(X, Y)$, then $T+S$ is a closed, almost upper semi-Fredholm linear relation and $\bar{k}(T+S)=\bar{k}(T)$.

(ii) If $T \in A L S F(X, Y)$ and $S^{\prime} \in S S\left(Y^{\prime}, X^{\prime}\right)$ then $T+S$ is a closed, almost lower semi-Fredholm linear relation and $\bar{k}(T+S)=\bar{k}(T)$.

Proof. We first note that by [3, Lemma 7], $T+S$ is closed. Furthermore, since $A L S F=F_{-}$, assertion (ii) is covered by Theorem 10(ii).

(i) By Lemma 12, $(X \times\{0\}, G(T)) \in A U S F$ and $(G(S), X \times\{0\}) \in S S$. Then it follows from Theorem 9 that the pair $(G(S), G(T))$ is almost upper semi-Fredholm, that is, the operator $Q_{G(T)} J_{G(S)}$ is injective and open. Moreover,

$\alpha\left(V Q_{G(T)} J_{G(S)} U\right) \leq \alpha(V)+\alpha\left(Q_{G(T)} J_{G(S)}\right)+\alpha(U)([21$, Lemma 5.1] $)=0($ Lemma 11) and

$\gamma\left(V Q_{G(T)} J_{G(S)} U\right) \geq \gamma(V) \gamma\left(Q_{G(T)} J_{G(S)}\right) \gamma(U)>0$ (by [7, Theorem II.3.11] and Lemma 11).

Now by Lemma 11(iii), we conclude that $T+S$ is almost upper semi-Fredholm so that $T+S$ is upper semi-Fredholm and this implies, by the use of Theorem 10(i) that $\bar{k}(T+S)=\bar{k}(T)$. The proof is complete.

ACKNOwledgements. This work was supported by MICINN (Spain), Grant MTM2010-20190.

\section{REFERENCES}

1. R. P. Agarwal, M. Meehan and D. O'Regan, Fixed point theory and applications (Cambridge University Press, Cambridge, UK, 2001).

2. T. Álvarez, On almost semi-Fredholm linear relations in normed spaces, Glasgow Math. J. 47 (2005), 187-193.

3. T. Álvarez, On the perturbation of semi-Fredholm relations with complemented ranges and null spaces, Acta Math. Sinica (English Series) 26 (2010), 1545-1554. 
4. T. Álvarez, R. W. Cross and D. Wilcox, Multivalued Fredholm-type operators with abstract generalised inverses, J. Math. Anal. Appl. 261 (2001), 403-417.

5. T. Álvarez, R. W. Cross and D. Wilcox, Quantities related to upper and lower semiFredholm-type linear relations, Bull. Austr. Math. Soc. 66 (2002), 275-289.

6. E. A. Coddington and A. Dijksma, Self-adjoint subspaces and eigenfunction expansions for ordinary differential subspaces, J. Differ. Equations 20 (1976), 473-526.

7. R. W. Cross, Multivalued linear operators (Marcel Dekker, New York, NY, 1998).

8. A. Dijksma, A. El Sabbah and H. S. V. de Snoo, Self-adjoint extensions of regular canonical systems with Stieltjes boundary conditions, J. Math. Anal. Appl. 152 (1990), 546-583.

9. A. Favini and A. Yagi, Multivalued linear operators and degenerate evolution equations, Ann. Mat. Pura. Appl. 163 (1993), 353-384.

10. D. Gheorghe, Erratum 'Stability of the index of a linear relation under compact perturbations' (Studia Math. 180 (2007), 95-102), Studia Math. 189 (2) (2008), 201-204.

11. D. Gheorghe, A Kato perturbation-type result for open linear relations in normed spaces, Bull. Austr. Math. Soc. 79 (2009), 85-101.

12. [12] S. Goldberg, Unbounded linear operators. Theory and applications (Dover, New York, NY, 1966).

13. M. González, Fredholm theory for pairs of closed subspaces of a Banach space, J. Math. Anal. Appl. 305 (2005), 53-62.

14. L. Gorniewicz, Topological fixed point theory of multivalued mappings (Kluwer, Dordrecht, Netherlands, 1999).

15. M. Gromov, Partial differential relations (Springer-Verlag, Berlin, Germany, 1986).

16. T. Kato, Perturbation theory for nullity, deficiency and other quantities of linear operators, J. d'Analyse Math. 6 (1958), 273-322.

17. L. E. Labuschagne, The perturbation of relatively open operators with reduced index, Proc. Math. Cambridge 112 (1992), 385-402.

18. M. Muresan, On a boundary value problem for quasi-linear differential inclusions of evolution, Collect. Math. 45 (1994), 165-175.

19. J. von Neumann, Functional operators II. The geometry of orthogonal spaces, Annals of Mathematics Studies, 22 (Princeton University Press, Princeton, NJ, 1950).

20. H. Román-Flores, A. Flores-Franulic, M. A. Rojas-Medar and R. C. Bassanezi, Stability of the fixed points set of fuzzy contractions, Appl. Math. Lett. 11 (1998), 33-37.

21. A. Sandovici and H. de Snoo, An index formula for the product of linear relations, Lin. Alg. Appl. 431 (2009), 2160-2171. 